\title{
The Role of Prolactin in Autoimmune Demyelination: Suppression of Experimental Allergic Encephalomyelitis by Bromocriptine
}

\author{
Peter N. Riskind, MD, PhD, Luca Massacesi, MD, Teresa H. Doolittle, PA-C, MHP, \\ and Stephen L. Hauser, MD
}

\begin{abstract}
Several lines of evidence suggest that the anterior pituitary hormone prolactin has a stimulatory role on immune function and that pharmacological suppression of prolactin secretion with the dopamine-agonist bromocriptine suppresses both humoral and cellular immunity. Here, we describe the effects of prolactin-suppression on the course of experimental allergic encephalomyelitis in female Lewis rats. Initiation of continuous bromocriptine treatment before immunization reduced both the severity and incidence of clinical signs of acute experimental allergic encephalomyelitis. Experimental allergic encephalomyelitis-immunized rats experienced a threefold rise in basal prolactin levels on day 4 after immunization and maintained elevated prolactin levels on day 10, before the onset of neurological signs of experimental allergic encephalomyelitis. Bromocriptine treatment reduced prolactin levels to those of shamimmunized rats. In vivo bromocriptine pretreatment inhibited splenic lymphocyte proliferative responses in vitro to the immunizing antigen and to concanavalin A. Moreover, bromocriptine therapy was protective when initiated 1 week after the initial immunization and was also effective in suppression of late disease. These results indicate that (1) prolactin levels are elevated after immunization and before the onset of experimental allergic encephalomyelitis, (2) bromocriptine inhibits both prolactin secretion and the severity of acute experimental allergic encephalomyelitis, and (3) inhibition is also present when treatment is begun after sensitization, suggesting an effect of prolactin on the effector limb of the immune response during experimental allergic encephalomyelitis.
\end{abstract}

Riskind PN, Massacesi L, Doolittle TH, Hauser SL. The role of prolactin in autoimmune demyelination: suppression of experimental allergic encephalomyelitis by bromocriptine. Ann Neurol 1991;29:542-547

Prolactin (PRL), the major hormonal stimulus to lactation, is a $24-\mathrm{kd}$ polypeptide hormone that is synthesized and secreted by the anterior pituitary gland. The importance of PRL to normal immune homeostasis was first shown by Nagy and Berczi [1], who found that hypophysectomized rats were severely immunocompromised, with profoundly depressed antibody titers to administered antigen and virtually absent delayed hypersensitivity responses. Surprisingly, in such animals, immunocompetence could be restored by exogenous PRL, and to a lesser extent by growth hormone, but not by other pituitary hormones [2]. Later work demonstrated that bromocriptine (BCR), a dopaminergic agonist that selectively inhibits PRL release, mimics the effect of hypophysectomy [3]. Treatment with PRL reversed the effect of $\mathrm{BCR}$, indicating that its activity was due to specific inhibition of PRL release.

Other studies indicated that excessive PRL levels may stimulate immune responses. PRI treatment of normal mice resulted in a dose-dependent increase in splenic cell responses to mitogens such as concanavalin A (ConA) [4, 5]. In rodent splenic lymphocytes, PRL also induced the expression of interleukin-2 receptors and promoted secretion of interferon gamma $[4,6]$. Sheep red blood cell responses were enhanced by PRL administered at the time of or 4 days after immunization, suggesting an effect on both the afferent and efferent limbs of the immune response [7]. These effects may be due to a direct action of PRL on lymphocytes as both $\mathrm{T}$ and $\mathrm{B}$ lymphocytes were found to have highaffinity receptors for PRL, and in the rat, the number of receptors per cell was similar for lymphocytes and mammary gland cells $[8,9]$. In studies of human lymphocytes, binding of the drug cyclosporine could be reversibly displaced by PRL, suggesting that cyclosporine-induced immunosuppression may be in part mediated by interaction with the PRL receptor [9].

Modulation of PRL secretion represents one potential approach to the control of disease states characterized by heightened immune reactivity, including auto-
From the Department of Neurology, Massachusetts General Hospital, Boston, MA.

Received Sep 4, 1990, and in revised form Nov 12. Accepted for publication Nov 19, 1990.
Address correspondence to Dr Riskind, Laboratory of Neuroendocrinology, CNY 6, Massachusetts General Hospital-East, Building 149, 13th Street, Charlestown, MA 02129. 
Table 1. The Effect of BCR Treatment Initiated 2 Days Before Immunization on the Course of Acute EAE

\begin{tabular}{|c|c|c|c|c|c|}
\hline Treatment & $\begin{array}{l}\text { Peak Clinical } \\
\text { EAE Score } \\
(\text { Mean } \pm \text { SEM })^{\mathrm{a}}\end{array}$ & $\begin{array}{l}\text { Neurological } \\
\text { Signs }\end{array}$ & $\begin{array}{l}\text { Day of Onset } \\
\text { (Mean } \pm \text { SEM) }\end{array}$ & $\begin{array}{l}\text { Duration } \\
\text { (Mean } \pm \text { SEM) }\end{array}$ & Deaths \\
\hline Control & $3.4 \pm 0.5$ & $10 / 11$ & $12.8 \pm 0.03$ & $5.5 \pm 0.7^{b}$ & $2 / 11$ \\
\hline BCR & $1.4 \pm 0.5^{c}$ & $5 / 11^{d}$ & $12.8 \pm 0.08$ & $4.8 \pm 0.5$ & $0 / 11$ \\
\hline
\end{tabular}

${ }^{a}$ Calculated as the highest EAE score for each rat during the acute illness. Unaffected rats received a score of zero.

${ }^{b}$ Excluding 2 rats that died during their acute illness.

$c_{p}<0.001$, Mann-Whitney $U$ test.

$d_{p}<0.05$, Fisher exact probability test.

Clinical scores were recorded daily by a blinded observer. Data are the results of two experiments, in each of which a treatment effect was observed ( $\mathrm{n}=11$ /group).

$\mathrm{BCR}=$ bromocriptine; $\mathrm{EAE}=$ experimental allergic encephalomyelitis.

immune diseases. In vitro, BCR has been shown to reduce lymphocyte responses to mitogens [4], antibody responses [7], and mixed lymphocyte reactivity [8]. In vivo, $B C R$ pretreatment inhibited graft versus host disease in rats [8], and a preliminary report indicated a possible effect of $B C R$ on experimental allergic encephalomyelitis (EAE) [3]. In the current report, we show that BCR treatment modifies the clinical course of acute rat EAE, inhibits the development of late relapses, and blocks the rise of PRL levels that is present in control immunized animals.

\section{Methods \\ Induction of $E A E$}

Adult (60-day-old) female, random-cycle Lewis rats (Charles River, Wilmington, MA, or Harlan Sprague Dawley, Madison, WI) were housed in a temperature-controlled $(22+$ $1^{\circ} \mathrm{C}$ ) and light-controlled room (lights on from 7 AM to 7 PM) with food and water freely available. Rats were immunized with $200 \mathrm{mg}$ of guinea pig spinal cord homogenate (GPSC) (Pel-Freez, Rogers, AR), previously frozen at $-80^{\circ} \mathrm{C}$, in complete Freund's adjuvant (CFA) supplemented with $1 \mathrm{mg} / \mathrm{ml} \mathrm{H} 37 \mathrm{Ra}$ (mycobacterium tuberculosis) or sham-immunized with an equal volume of sterile saline. Bilateral intradermal injections were made over the dorsum, in accordance with the recommendations of the Subcommittee for Animal Care at Massachusetts General Hospital (Charlestown, MA).

Clinical EAE scores were graded daily by the following standard scoring system: 0 , no neurological signs; 1 , diminished tone and paresis of the tail; 2, weak hind limbs; 3 , plegic hind limbs; 4, quadriparesis; and 5, death. A value of 0.5 was added for incontinence. Rats were graded according to the highest single score attained, without addition of scores.

\section{$B C R$ Treatment}

Rats were lightly anesthetized with ether for subcutaneous implantation of a single pellet containing either BCR or a control pellet (Innovative Products of America, Toledo, $\mathrm{OH})$. The BCR pellets were designed to continuously release the drug at a rate of $2.3 \mathrm{mg} / \mathrm{day}$ for 21 days [4]. Control pellets contained the same carrier binder as the BCR pellets (cholesterol, stearate, phosphates, $\alpha$-lactose, and other proprietary ingredients).

\section{Measurement of Prolactin Levels}

Serum PRL levels were measured from trunk blood and from pituitary extracts in duplicate by radioimmunoassay (RIA), using materials provided by the National Institute of Diabetes, Digestive and Kidney Diseases, as previously reported [10]. Pituitary extracts were prepared by sonicating each pituitary in $1 \mathrm{ml}$ of $0.65 \mathrm{M}$ borate buffer, $\mathrm{pH} 9.0$, centrifugation at $15,000 \mathrm{~g}$ for 10 minutes, and decantation of the supernatant. The sensitivity of the RIA is less than $0.25 \mathrm{ng} / \mathrm{ml}$. Intra-assay and interassay coefficients of variation are less than $5 \%$.

\section{Proliferative Assays}

Spleens from killed rats ( $\mathrm{n}=7 /$ group) were aseptically removed and mechanically dispersed. Splenic lymphocytes were pooled and isolated by Ficoll-gradient centrifugation (Pharmacia, Piscataway, NJ). Cells were plated in triplicate in 96-well plates at a concentration of $2 \times 10^{5}$ cells/well, with medium consisting of RPMI 1640 (GIBCO, Grand Island, NY), suppiemented with $5 \%$ heat-inactivated fetal calf serum (GIBCO), 1\% L-glutamine, $1 \%$ NaPyruvate (GIBCO), 1.25\% HEPES buffer (GIBCO), 1\% nonessential amino acids, $1 \%$ penicillin-streptomycin (GIBCO), and $5 \times$ $10^{-5} \mathrm{M} 2$-mercaptoethanol. Additionally, GPSC $(0.1 \%)$ or ConA $(5 \mu \mathrm{g} / \mathrm{ml}$ ) (Sigma Chemical, St Louis, MO) were included in the medium. After 80 hours of incubation, $\left[{ }^{3} \mathrm{H}\right]$ rhymidine was added for another 16 hours of incubation, cells were harvested, and thymidine incorporation was measured in a scintillation counter. The proliferative response was expressed as counts per minute (cpm), and as stimulation indices (SI), defined as the stimulated culture counts per minute divided by the unstimulated culture counts per minute.

\section{Results}

\section{Bromocriptine Therapy Moderates Acute EAE}

Typical signs of EAE appeared in nearly $100 \%$ of immunized (but otherwise untreated) rats between day 12 and day 16 after immunization. A clinical EAE score of 3 or greater was present in most rats and was followed by gradual recovery within 1 week (Table 1). 


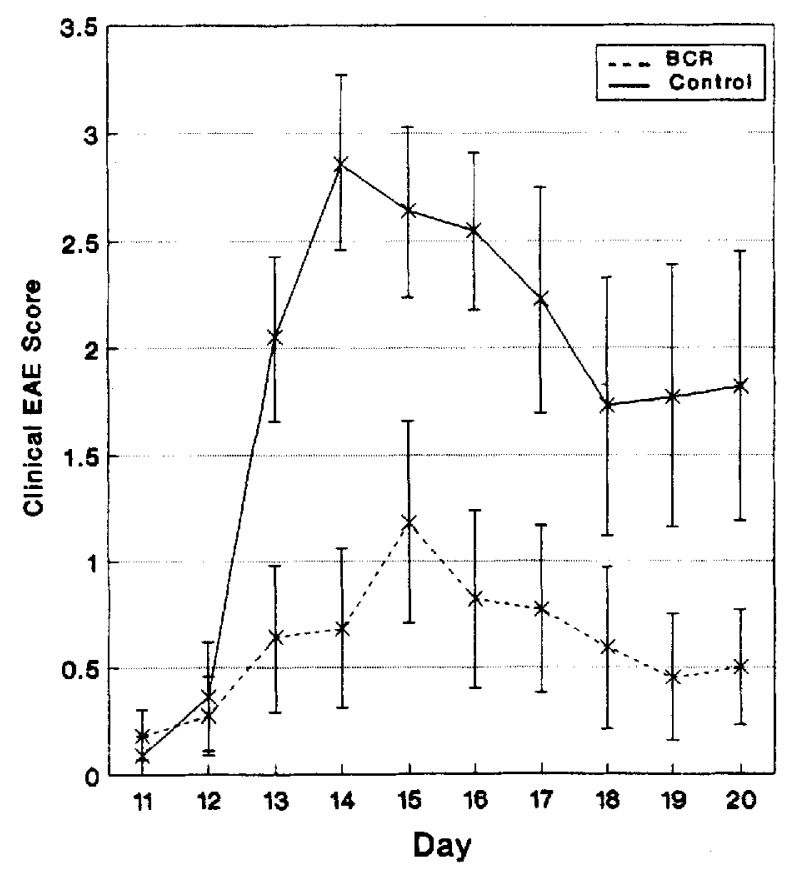

The effect of bromocriptine ( $B C R$ ) pretreatment on the severity of experimental allergic encephalomyelitis (EAE). Rats were subcutaneously implanted with a continuous-release BCR pellet (2.3 mg/day for 21 days) (dashed line) or a control pellet (solid line) 2 days before immunization ( $n=11 /$ group). The day after immunization appears on the $X$ axis, and the corresponding mean clinical EAE score ( $\pm S E M$ ) appears on the $Y$ axis. Clinical EAE scores were graded daily by a blinded investigator.
The effect on EAE of BCR pellets implanted 2 days before immunization is shown in the Figure and in Table 1. When compared with dummy-implant controls, BCR treatment reduced both the incidence of signs of $\mathrm{EAE}(p<0.05$, Fisher exact probability test) as well as the clinical EAE score $(p<0.001$ MannWhitney $U$ test). Two BCR-treated rats had signs of disease beginning 1 day after exhaustion of the BCR pellet. One of these rats had not had previous clinical signs of EAE.

Proliferative studies of splenic lymphocytes harvested from rats killed on day 10 after immunization revealed a pronounced reduction in mitogenic responses both to the immunogen and to ConA in BCRtreated rats (Table 2).

Immunization was associated with a greater than threefold rise in serum PRL levels (Table 3). BCR treatment effectively inhibited this rise in serum and reduced pituitary PRL content measured 10 days after immunization.

\section{Delayed Initiation of Bromocriptine Treatment Moderates Acute and Late Disease}

$B C R$ pellets implanted 1 week after immunization were also effective in moderating the incidence of signs of acute $\operatorname{EAE}\left(p<0.01, \chi^{2}\right)$ and the peak clinical EAE score $(p<0.025$, Mann-Whitney $U$ ) (Table 4). Additionally, a reduction in the incidence and severity of late disease was found in the BCR-treatment compared with the control-treatment group $(p<0.025)$ (see Ta-

Table 2. Proliferative Responses of Pooled Splenic Lymphocytes from Control or BCR-Pellet-Implanted Rats $(n=7 / G$ roup)

\begin{tabular}{lccl}
\hline Treatment & Background & $0.1 \%$ GPSC & ConA $(5 \mu \mathrm{g} / \mathrm{ml})$ \\
\hline Control & $750 \pm 100$ & $13,500 \pm 800($ SI $=18)$ & $283,000 \pm 24,000($ SI $=377)$ \\
BCR & $1,700 \pm 240$ & $8,600 \pm 1,800($ SI $=5.1)$ & $149,000 \pm 36,000($ SI $=87.6)$
\end{tabular}

Pellets were implanted 2 days before immunization for EAE and splenic lymphocytes harvested on day 10 after immunization. Results are expressed as counts per minute (mean \pm SEM) and as stimulation indices (SI) to guinea pig spinal cord homogenate (GPSC) or to Concanavalin A (ConA). See text for details.

$\mathrm{BCR}=$ bromocriptine; $\mathrm{EAE}=$ experimental allergic encephalomyelitis.

Table 3. Prolactin Levels (Mean $\pm S E M)$ in Lewis Rats Immunized for $E A E(n=7 / G$ roup)

\begin{tabular}{llll}
\hline Group & Day $4^{\mathrm{a}}$ Serum $(\mathrm{ng} / \mathrm{ml})$ & Day $10^{\mathrm{b}}$ Serum $(\mathrm{ng} / \mathrm{ml})$ & Pituicary $(\mu \mathrm{g} / \mathrm{gland})$ \\
\hline Sham immunization & $27 \pm 13$ & $\ldots$ & $\ldots$ \\
Immunization & $109 \pm 37^{\mathrm{c}}$ & $\ldots$ & $\ldots$ \\
$\quad$ Control & $\ldots$ & $>125$ & $126 \pm 14$ \\
BCR & $\ldots$ & $36 \pm 12^{\mathrm{c}}$ & $97 \pm 12$ \\
\hline
\end{tabular}

${ }^{a}$ Serum PRL measurement 4 days after immunization.

${ }^{b}$ Serum level and pituitary content of PRL 10 days after immunization.

${ }^{c} p<0.01$.

$\mathrm{EAE}=$ experimental allergic encephalomyelitis; $\mathrm{BCR}=$ bromocriptine; $\mathrm{PRL}=$ prolactin. 
Table 4. Effect of Late Initiation of BCR Treatment on the Course of EAE

\begin{tabular}{|c|c|c|c|c|c|c|c|c|}
\hline \multicolumn{4}{|c|}{ Acute EAE } & \multicolumn{5}{|c|}{ Late Disease } \\
\hline Treatment & $\begin{array}{l}\text { Peak Clinical } \\
\text { EAE Score } \\
(\text { Mean } \pm \text { SEM })^{a}\end{array}$ & $\begin{array}{l}\text { Neurological } \\
\text { Signs }\end{array}$ & Deaths & $\begin{array}{l}\text { Peak Clinical } \\
\text { EAE Score } \\
(\text { Mean } \pm \text { SEM })^{a}\end{array}$ & $\begin{array}{l}\text { Neurological } \\
\text { Signs }\end{array}$ & $\begin{array}{l}\text { Day of Onset } \\
\text { (Mean } \pm \text { SEM) }\end{array}$ & $\begin{array}{l}\text { Duration } \\
\text { (Mean } \pm \text { SEM) }\end{array}$ & Deaths \\
\hline Control & $1.7 \pm 0.3$ & $13 / 15$ & 0 & $2.9 \pm 0.6$ & $8 / 8$ & $22.4 \pm 0.5$ & $6 \pm 0.6^{b}$ & 2 \\
\hline $\mathrm{BCR}$ & $0.7 \pm 0.3^{\mathrm{c}}$ & $7 / 17^{\mathrm{d}}$ & 0 & $1.2 \pm 0.4^{\mathrm{c}}$ & $4 / 8^{c}$ & $22.6 \pm 0.7$ & $5 \pm 0.6$ & 0 \\
\hline
\end{tabular}

Includes zero scores for unaffected rats.

bxcludes 2 rats that died during the late relapse.

${ }^{c} p<0.025$, Mann-Whitney $U$ test, Fisher exact probability test.

${ }_{p} p<0.01, \chi^{2}$ test

Acute EAE was assessed in rats in which BCR or control pellets were implanted 7 or 8 days after immunization. Late disease was assessed in the rats that were treated on day 8 after immunization.

$\mathrm{BCR}=$ bromocriptine; $\mathrm{EAE}=$ experimental allergic encephalomyelitis.

ble 4). It was again observed that 2 of $8 \mathrm{BCR}$-treated rats developed signs of late disease beginning 1 and 3 days after exhaustion of the BCR pellet.

\section{Discussion}

These results indicate that BCR pretreatment of susceptible Lewis rats resulted in substantial protection from acute EAE, and that protection was associated with a proportional decrease in splenic proliferative responses to both immunogen and to ConA. BCR treatment initiated 1 week after immunization, at a time after sensitization had occurred [11], also inhibited acute EAE and, in addition, prevented late disease that typically occurs in female rats during the early recovery period $[12,13]$. Moreover, signs of EAE developed in several rats after the BCR pellets were exhausted. These results indicate that BCR protection results at least in part from inhibition of the effector limb of the T-cell response in rat EAE.

Protection was incomplete because nearly $50 \%$ of BCR-treated, immunized rats developed signs of EAE that were generally mild and consisted of a loss of tail tone or an unsteady gait. It is possible that higher dosages or more effective delivery of BCR might provide more complete inhibition of disease. This is suggested by the finding that suppressed but detectable serum PRL levels were present in BCR-treated rats. Additionally, the effects of BCR on the pathology of EAE at different stages during the disease course is interesting, and these studies are underway.

It is likely that the effects of BCR in rat EAE are mediated by inhibition of PRL secretion. PRL suppression achieved by other routes, for example, by the nondopaminergic drug cysteamine or by hypophysectomy, also results in functional immunosuppression [1-5]. BCR does not bind rat lymphocytes [14], and the immunosuppressive properties of this drug are reversed by the administration of PRL [3-5].

Previous work in other disease models also indicates that BCR might moderate ongoing autoimmunity.
When mice are immunized with bovine type II collagen in CFA 4 to 8 weeks before pregnancy, they develop a high frequency of autoimmune arthritis during the postpartum period; in some mice, arthritic signs resolve during subsequent pregnancies and recrudesce after delivery [15]. Postpartum BCR treatment suppresses the clinical exacerbarion of postpartum joint disease [15], consistent with the hypothesis that lactational elevation of PRL levels contributes to recurrent arthritis months after the initial immunization.

Under normal conditions, PRL is secreted episodically, in pulses of varying size and duration, with highest levels during the night in association with sleep [16]. In addition to its underlying diurnal secretory rhythm, PRL is acutely secreted in response to stress $[16,17]$. In contrast to the effects of acure stress, chronic stress (or chronic adrenocorticoid treatment) inhibits PRL secretion [18]. PRL is secreted in large amounts during the postpartum period, and is the most important hormonal stimulus for lactation in mammals $[10,16]$. It might be predicted that in immunized animals, "stress" hormones such as adrenocorticotropic hormone and PRL would be erratically stimulated as a result of nonspecific discomfort. Besedovsky and associates [19] and Saphier [20], however, have demonstrated that a coordinated partern of hypothalamic neurochemical and neuroendocrine events occurs in concert with other aspects of the immune response; for example, hypothalamic noradrenergic activity is inhibited at the peak of the immune response to sheep red blood cells, several days before the rise of plasma corticosterone levels.

Neuroendocrine responses also occur in a stereotypical, predictable pattern in EAE; corticosterone levels rise predictably just before the onset of paralysis, reaching peak levels at the time of maximum neurological disease [21]. The rise of corticosterone is an essenrial homeostatic response because adrenalectomized animals invariably die within a few days of immunization. Adrenalectomy soon after the onset of EAE pro- 
motes EAE relapses [22], suggesting that release of endogenous glucocorticoids modulates both the initial and the recurrent phases of the disease. In the current experiments, the severity of EAE was reduced in rats implanted with control pellets 1 week after immunization, perhaps due to effects of acute corticosterone secretion imparted by ether anesthesia.

There is little information regarding PRL secretion during immune responses, and thus, the current findings that serum PRL levels are elevated in rat EAE are particularly interesting. One recent report indicated that PRL levels of male rats are stimulated to $150 \%$ of control levels during the first 6 days after immunization with $\mathrm{CFA} / M$. bovis [23]. In $\mathrm{EAE}$, the rise in serum PRL is likely a consequence of immunization and is not disease specific, but further studies will be required to clarify this point. One potential mechanism of PRL stimulation during immune responses involves lymphokine-mediated stimulation of PRL secretion through direct actions on the hypothalamus or pituitary [24].

In humans, elevated serum PRL levels have been reported to follow cardiac transplantation and to be present in some patients with chronic idiopathic iridocyclitis $[25,26]$. In the former condition, a high level of circulating PRL was believed to presage graft rejection, and in the latter, a clinical response to BCR has been claimed. Previous studies indicate that there is an increased risk of attacks of multiple sclerosis in the postpartum period, a time of physiological PRL hypersecretion [27-34]. Other autoimmune diseases have been observed to occur almost uniquely (e.g., lymphocytic hypophysitis) or more commonly (rheumatoid arthritis and myasthenia gravis) during the puerperium $[35,36\}$. Although the neuroendocrine regulation of immune homeostasis is undoubtedly complex, inhibition of PRL secretion may thus represent a potential approach to the control of autoimmune disorders.

This work was supported by a grant from the National Multiple Sclerosis Society (NMSS), by a gift from the Fidia Pharmaceutical $\mathrm{Co}$, and by grants from the Mathers Foundation and the Dynatech Corporation. S. L. H. is a Harry Weaver Neuroscience Fellow of the NMSS.

\section{References}

1. Nagy E, Berczi I. Immunodeficiency in hypophysectomized rats. Acta Endocrinol (Copenh) 1978;89:530-537

2. Nagy E, Berczi I, Fricsen HG. Regulation of immunity in rats by lactogenic and growth hormone. Acta Endocrinol (Copenh) 1983;102:351-357

3. Nagy E, Berczi I, Wren GE, et al. Immunomodulation by bromocriptine. Immunopharmacology 1983;6:231-243

4. Bernton EW, Meltzer MS, Holaday JW. Suppression of macrophage activation and T-lymphocyte function in hypoprolactinemic mice. Science 1988;239:401-404
5. Bernton EW. Prolactin and immune host defenses. PNEI Perspect 1989;2:21-29

6. Mukherjee P, Mastgro AM, Hymer WC. Prolactin induction of interleukin-2 receptors on rat splenic lymphocyres. Endocrinology $1990 ; 126: 88-94$

7. Spangelo BL, Hall NRS, Ross PC, Goldstein AL. Stimulation of in vivo antibody production and concanavalin- $A$-induced mouse spleen cell mitogenesis by prolactin. Immunopharmacology $1987 ; 14: 11-20$

8. Hiestand PC, Mekler P, Norddmann R, et al. Prolactin as a modulator of lymphocyte responsiveness provides a possible mechanism of action for cyclosporine. Proc Natl Acad Sci USA 1986;83:2599-2603

9. Russell DH, Kibler R, Matrisian L, et al. Prolactin receptors on human $\mathrm{T}$ and $\mathrm{B}$ lymphocytes: antagonism of prolactin binding by cyclosporine. J Immunol 1985;134:3027-3031

10. Riskind PN, Millard WJ, Martin JB. Evidence that thyrotropinreleasing hormone is not a major prolactin-releasing factor during suckling in the rat. Endocrinology 1984;115:312-316

11. Steinman L, Rosenbaum JT, Sriram S, McDevitt HO. In vivo effects of antibodies to immune response gene products: prevention of experimental allergic encephalitis. Proc Natl Acad Sci USA 1981;78:7111-7114

12. McFarlin DE, Blank SE, Kibler RF. Recurrent experimental allergic encephalomyelitis in the Lewis rat. J Immunol 1974; 113:712-715

13. Keith AB. Sex difference in Lewis rats in the incidence of recurrent experimental allergic encephalomyelitis. Nature 1978; 272:824-825

14. Ovadia $\mathrm{H}, \dot{A}$ bramsky $\mathrm{O}$. Dopamine receptors on isolated membranes of rat lymphocytes. J Neurosci Res 1987;18:70

15. Whyte $A$, Williams RO. Bromocriptine suppresses postpartum exacerbation of collagen-induced arthritis. Arthritis Rheum 1988;31:927-928

16. Negro-Vilar A, Lopez F. Neuroendocrine regulation of prolactin secretion. PNEI Perspect 1989;2:11-16

17. Neil JD. Effect of "stress" on serum prolactin and luteinizing hormone levels during the estrous cycle of the rat. Endocrinology 1970;87:1192-1197

18. Fang VS, Shian LR. Adrenal influence on pituitary secretion of rhyrotropin and prolactin in rats. Endocrinology 1981;108: $1545-1551$

19. Besedorsky H, del Rey A, Sorkin E, et al. The immune response evokes changes in brain noradrenergic neurons. Science 1983;221:564-565

20. Saphier D. Neurophysiological and endocrine consequences of immune activity. Psychoneuroendocrinology 1989;14:63-87

21. Macphee IAM, Antoni FA, Mason DW. Spontaneous recovery of rats from experimental allergic encephalomyelitis is dependent on regulation of the immune system by endogenous adrenal corticosteroids. J Exp Med 1989;169:431-445

22. Levine $S$, Sowinski $\mathbf{R}$, Steinetz B. Effects of experimental allergic encephalomyelitis on thymus and adrenal: relation to remission and relapse. Proc Soc Exp Biol Med 1980;165:218-220

23. Neidhart M. Bromocriptine microcapsules inhibit ornithine decarboxylase activity induced by Freund's complete adjuvant in lymphoid tissues of male rats. Endocrinology 1989;125: 2846-2852

24. Spangelo BL, Judd AM, Isakson PC, MacLeod RM. Interleukin- 6 stimulates anterior pituitary hormone release in vitro. Endocrinology 1989;125:575-577

25. Carrier M, Emery RW, Wild-Mobley JW, et al. Prolactin as a marker of rejection in human heart transplantation. Transplant Proc 1987;19:3442-3443

26. Hedner PL, Bynke G. Endogenous iridocyclitis relieved during treatment with bromocriptine. Am J Ophthalmol 1985;100: 618-619 
27. Millar JHD, Allison RS, Cheeseman EA, Merett JD. Pregnancy as a factor influencing relapse in disseminated sclerosis. Brain 1959;82:417-426

28. Shapira K, Poskanzer DC, Newell DJ, Miller H. Marriage, pregnancy and muitiple sclerosis. Brain 1966;89:419-428

29. Leibowitz UA, Antonovsky A, Kats R, Alter M. Does pregnancy increase the risk of multiple sclerosis? J Neurol Neurosurg Psychiatry 1967;30:354-357

30. Ghezzi A, Caputo D. Pregnancy: a factor influencing the course of multiple sclerosis? Eur Neurol 1981;20:517-519

31. Korn-Lubetzki I, Kahana E, Cooper G, Abramsky O. Activity of multiple sclerosis during pregnancy and puerperium. Ann Neurol 1984;16:229-231
32. Thompson DS, Nelson LM, Burks A, et al. The effects of pregnancy in multiple sclerosis: a retrospective study. Neurology 1986;36:1097-1099

33. Birk K, Rudich R. Pregnancy and multiple sclerosis. Arch Neurol 1986;43:719-726

34. Birk K, Smeltzer S, Ford C, et al. The effect of pregnancy on multiple sclerosis. Neurology 1988;38(suppl 1):237 (Abstract)

35. Baskin DS, Townsend JJ, Wilson CB. Lymphocytic hypophysitis of pregnancy simulating a pituitary adenoma: a distinct pathological entity. J Neurosurg 1982;56:148-153

36. Lahita RG. Sex steroids and the theumatic diseases. Arthritis Rheum 1985;28:121-126 